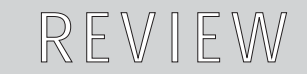 \\ Mycobacterium avium-intracellulare infection during HIV disease. Persisting problems
}

\section{Roberto Manfredi}

Department of Infectious Diseases, “Alma Mater Studiorum” University of Bologna, S. Orsola-Malpighi Hospital, Bologna-Italy

Key words: HIV disease, Opportunistic infections, Combined antiretroviral therapy, Mycobacterium avium-intracellulare, Diagnosis, Treatment, Prophylaxis

Infezioni da Mycobacterium avium-intracellulare in corso di malattia da HIV. Problematiche attuali

\section{SUMMARY}

Still in the era of combined antiretroviral therapy, late recognition of HIV disease or lack of sufficient immune recovery pose HIV-infected patients at risk to develop opportunistic infections by nontuberculous mycobacteria (NTM), which are environmental organisms commonly retrieved in soil and superficial waters. Among these microorganisms, the most frequent is represented by Mycobacterium avium complex (MAC). Health care professionals who face HIV-infected patients should suspect disseminated mycobacterial disease when a deep immunodeficiency is present, (a CD4+ lymphocyte count below 50 cells/ $\mu \mathrm{L}$ ) often associated with constitutional signs and symptoms, and non-specific laboratory abnormalities. Mycobacterial culture of peripheral blood is a reliable technique for diagnosing disseminated disease. Among drugs active against NTM, as well as some anti-tubercular compounds, the rifampin derivative rifabutin, and some novel fluoroquinolones, the availability of macrolides, has greatly contributed to improve both prophylaxis and treatment outcome of disseminated MAC infections. Although multiple questions remain about which regimens may be regarded as optimal, general recommendations can be expressed on the ground of existing evidences. Treatment should begin with associated clarithromycin (or azithromycin), plus ethambutol and rifabutin (with the rifabutin dose depending on other concomitant medications that might result in drug-drug interactions). A combined three-drug regimen is preferred for patients who cannot be prescribed an effective antiretroviral regimen immediately. Patients with a CD4+ lymphocyte count below 50 cells $/ \mu \mathrm{L}$, who do not have clinical evidence of active mycobacterial disease, should receive a primary prophylaxis with either clarithromycin or azithromycin, with or without rifabutin.

\section{Received June 9, 2008}

\section{INTRODUCTION}

Mycobacterium avium-intracellulare complex (MAC) consists of acid-fast microorganisms ubiquitous in the environment that are an infrequent cause of disease in individuals with normal immune defence. In deeply immunocompromised patients, such as those with advanced HIV infection-AIDS, MAC still represent common and serious opportunistic infections (12, 44). Among HIV-infected subjects followed in the pre-antiretroviral therapy era, disseminated MAC has historically occurred when the CD4+ T-lymphocyte count dropped at values $<50$ cells/ $\mu \mathrm{L}(12,44)$. Colonization of the airways (and also the gastrointestinal tract) by MAC can occur without evident morbidity; however, MAC colonization of these sites underlines that such patients are at increased risk for developing disseminated MAC infection

\section{Accepted August 4, 2008}

(13). The combination antiretroviral therapies (cART) introduced since mid-1996, have been associated with a significant reduction in AIDSrelated mortality, days of hospitalization, and the incidence of recurring opportunistic infections, including MAC disease (48). However, since the introduction of cART $(3,29,41,50,52,57)$, there have been numerous reports of atypical MAC clinical presentations, so that diagnosis remains still difficult. The diagnosis can pose challenges to the clinician because isolation of these organisms is often difficult. In addition, both active disease and airway colonization occur in individuals with structural defects, previous or concurrent illnesses, adding further confusion for health caregivers. Furthermore, these organisms are frequently contaminants, leading to false-positive diagnoses in patients with other active diseases. The treatment

\section{Corresponding author: Roberto Manfredi}

Universiy of Bologna- c/o Infectious Diseases, S. Orsola Hospital

Via Massarenti II - 40 I 38 Bologna, Italy - Telephone: +39-05I-6363355; Telefax: +39-05I-343500

E-mail: roberto.manfredi@unibo.it 
of non-tuberculous mycobacteria (NTM) infections is as difficult as establishing a correct diagnosis because therapy is often prolonged, difficult to tolerate, and frequently ineffective. In this context, clinicians should be familiar with these emerging disorders, whose incidence may increase among immunocompromised patients other than those with HIV/AIDS (12). There is convincing evidence that an effective treatment of disseminated MAC infection improves both survival and quality of life of patients with HIV infection (55). Whether MAC can be eradicated once disseminated infection has occurred is still uncertain, but some evidences suggest that secondary prophylaxis may be discontinued in patients who have had sustained immunologic recovery further to an effective cART $(1,2,80)$.

\section{Microbiological and epidemiological notes}

Mycobacterium avium-intracellulare complex (MAC) consists of four recognized species (Mycobacterium avium, Mycobacterium intracellulare, Mycobacterium chimerae, Mycobacterium colombiense) and several unnamed organisms not belonging to any of these taxa referred to as cluster $\mathrm{X}$ or MAIX. In the past, it was difficult to distinguish $M$. avium from $M$. intracellulare, as species identification relied on biochemical tests and serotyping. Therefore, it was common to refer to them as $M$ avium-intracellulare. Of the 28 known seroagglutination MAC types, types 1 to 6 , types 8 to 11, and type 21 are classified as M. avium; type 7, types 12 to 20, and type 25 are classified as $M$. intracellulare; while types 22 to 24 and types 26 to 28 have not been classified until recent years (60). Using DNA probe techniques, it is now possible to rapidly identify $M$. avium and $M$. intracellulare (39). However, in most cases of AIDS-related disease, disseminated MAC infection is caused by $M$. avium species, and from a therapeutic-prophylactic point of view no significant difference occur. MAC strains may vary in their pathogenicity, which may explain why M. avium serotypes 4 and 8 are the most common organisms to infect immunocompromised patients suffering from AIDS (61, 77). Some identified biological markers of MAC pathogenicity include the presence of plasmid, antibiotic susceptibility patterns, multilocus enzyme electrophoresis profiles, and restriction fragment length polymorphism patterns (16, 26, 30). Furthermore, changes in colony morphology may play a role in influencing the virulence of some NTM species, with special focus on MAC, with smooth versus rough morphotypes potentially associated with more virulent, invasive clinical pictures $(33,37)$. Superficial water, soil and dust have been implicated as possible environmental sources of MAC for HIV-infected patients (as in the general population). During a documented nosocomial outbreak, the hospital water supply was the ascertained origin of a well defined MAC strain, with showed the same restriction fragment length polymorphism profile as that infecting a group of hospitalized AIDS patients (66). Furthermore, no apparent differences have been observed in the frequency of disseminated MAC infection according to age, gender, race, geographic areas, the different exposure to HIV infection transmission, antiretroviral therapy selection, and initial AIDS-defining conditions (32).

\section{Pathogenesis and pathologic features}

The mechanisms of spread and acquisition of disseminated MAC infection are still incompletely understood. As such, not all NTM isolates represent true infections, since they may be trivial colonization or contamination. Currently available data support the hypothesis that MAC disease results from recent environmental transmission through either the respiratory or the gastrointestinal tract, rather than from a reactivation of latent MAC infection. Should reactivation of latent infection occur as the prevalent, it would be expected to increase with age, like in tuberculosis. On the contrary, among HIV-infected patients with a primary disease manifestation, the occurrence of MAC dissemination does not vary within different age groups (32). Anyway, after acquiring a clinically evident MAC infection, underlying immunodeficiency becomes the most relevant factor, which supports disease recurrences during the follow-up. From an immunological point of view, the risk of developing HIV-associated MAC infection is inversely related with the absolute CD4+ lymphocyte count. In one observational study, the measured one-year incidence of MAC bacteremia was $3 \%$ for patients with a CD4+ count ranging from 100 to 199 cells/ $\mu \mathrm{L}$, as compared with $39 \%$ for patients with a CD4+ count $<10$ cells $/ \mu L$ (44). The same study also underlined that individuals with a far advanced HIV disease had a linear increase in the risk of developing MAC bacteremia over time, and the risk of developing disseminated MAC infection for patients surviving for 30 months after being diagnosed with AIDS was around 50\% (44). Other observational studies and prophylaxis trials added several, consistent findings: the risk of developing a disseminated MAC disease ranged from $30 \%$ to $50 \%$ in patients with CD4+ lymphocyte counts $<50$ cells $/ \mu \mathrm{L}$ which did not undergo specific antimicrobial prophylaxis (12, 45, 51). In particular, the difficulty to demonstrate a retrievable antibody response to MAC in HIV-infected patients with disseminated MAC infection may 
also suggests a recent primary infection, because deeply immunocompromised HIV-infected patients lack the ability to establish antibody responses to multiple, primary infections $(69,70)$. Furthermore, literature reports of MAC colonization of the respiratory or gastrointestinal tract preceding the occurrence of disseminated MAC infection suggest that the organism is first acquired from the environment and subsequently disseminated, when a severe underlying immunosuppression is present $(13,36)$. Finally, the incidence of disseminated MAC disease among patients with AIDS substantially exceeds the frequency of MAC infection in the general population, as determined by population studies, and confirmed also by MAC-specific skin testing (65). By the year 1984, only 37 cases of disseminated MAC infection in non-HIV-infected humans were reported, the majority of them in patients with identifiable, severe immunological deficiencies (31). In the non-HIV population, MAC infection generally caused localized lesions: cervical lymphadenitis in children, a slowly progressive fibrocavitary pulmonary disease in middle-aged men with a chronic, underlying lung disease, or localized bronchiectasis in women who had pectum excavatum, scoliosis, or mitral valve prolapse and associated conditions (i.e. Marfan's syndrome) $(35,71)$. The blunted immune response and the lack of local and systemic inflammatory reaction typical of patients with AIDS and a deep HIV-related immunodeficiency who develop disseminated MAC disease is consistent with the hypothesis that the key immunologic defects associated with dissemination relies on an unpaired monocyte-macrophage killing of mycobacteria and a defective cytokine network response, including abnormally low levels of tumor necrosis factor (TNF), interferon-gamma, and interleukin-12 $(4,9,18,42,79)$. As a result of this extremely permissive immunological environment, MAC infection becomes widespread in extremely immunosuppressed HIV-infected patients, and it was the case of most subjects suffering from MAC disease during the pre-cART era. In the majority of autopsy examinations performed in patients who died of MAC infection in the pre-cART era, mycobacteria have been isolated from lymph nodes, spleen, liver, lung, adrenal glands, gastrointestinal tract, kidney, and bone marrow, but multiple dissemination has virtually no limits, and may involve all body organ-sites. In fact, the magnitude of mycobacteremia has been estimated to be as high as 10,000 organisms $/ \mu \mathrm{L}$ of blood, the equivalent of 10 billion organisms per gram of bone marrow, spleen, lymph node, and liver tissue obtained at necropsy examination (72). Not surprisingly, the histopathologic examination of liver, spleen, bone marrow, or gut from patients with AIDS-related disseminated MAC disease shows a high-grade widespread infection, together with a lack (or reduced) inflammatory infiltrate or tissue necrosis. In contrast, histopathologic examination of tissue from patients with AIDS who have localized MAC infection demonstrates marked inflammatory reaction and tissue destruction similar to the histopathologic findings observed in association with non-HIV MAC infections. On the other hand, the rapid recovery of immune defences obtained following cART introduction, is the basis of the so-called immune-reconstitution syndrome, which is more prominent just in patients with a prior-latent, or a recently acquired MAC infection.

\section{Clinical Presentation}

Subjects suffering from a disseminated MAC infection frequently show non-specific signs-symptoms, and multiple laboratory abnormalities. In many instances, a progressive worsening of constitutional symptoms may reflect a disseminated MAC infection, and this aspect can be incorrectly referred as to the progression of HIV disease itself. Patients affected by MAC disease most commonly report persistent fever (not responding to a broadspectrum empiric antibiotics), night sweats, asthenia, fatigue, anorexia, and weight loss up to cachexia (the so-called wasting syndrome). Non specific abdominal pain or chronic diarrhoea, may result from the involvement of retroperitoneal lymphnodes or gastrointestinal mucosa, respectively, by disseminated MAC disease A considerable liver and lymphnodes enlargement as demonstrated by abdominal computed tomography (CT) and ultrasonographic studies may also complete the clinical picture (47). From a laboratory point of view, pancytopenia is the most common occurrence, while elevated serum alkaline phosphatase and lactic dehydrogenase levels, or low albumin levels have been also reported. In a prospective, observational survey of MAC bacteremia conducted at a singlecentre US Hospital during the pre-cART era, the Authors interviewed patients with a CD4+ count $<50$ cells $/ \mu \mathrm{L}$ regarding signs and symptoms, and reviewed the laboratory workup conducted as part of the diagnostic evaluation for disseminated MAC infection (13). A history of fever lasting for more than one month, a hematocrit under $30 \%$, or serum albumin levels below $3.0 \mathrm{~g} / 100 \mathrm{ml}$, proved affordable predictors of MAC bacteremia. On the other hand, fatigue, diarrhoea, weight loss, neutropenia, and thrombocytopenia did not seem to play a significant role in discriminating between patients who were subsequently found to have MAC-positive blood cultures, and those who were culture-negative for MAC (13). More unusual 
manifestations of visceral MAC infection include a broad spectrum of localizations, including oral (palatal-gingival) ulcerations, septic arthritis, osteomyelitis, endophthalmitis, pericarditis, and gastrointestinal bleeding $(7,8,14,64,73)$. NTM lung infection is almost always associated with symptoms such as chronic or recurring cough, sputum production, and exertional dyspnoea. Although uncommon, haemoptysis can occur, thus entering tuberculosis in the differential diagnosis. However, also in patients with a suspected respiratory localization, constitutional symptoms occur, and often predominate.

\section{Diagnosis and microbiological workup}

The diagnosis of disseminated MAC disease frequently poses severe challenges to the clinicians. As recovery of these microorganisms may represent airway colonization, rather than true infection, diagnosis must be based on the compatibility between a high clinical suspicion (symptoms, clinical features, imaging) and microbiological findings. When a respiratory localization is suspected, sputum cultures are not reliable since they are often negative, and bronchial washing (or preferably BAL) samples, may be a more useful diagnostic tool. According to the American Thoracic Society criteria (25), a single positive culture from bronchial washing or BAL specimens may be considered diagnostic. Tissue fragments obtained from biopsy studies (transbronchial lung biopsy, surgical lung biopsy, or excision biopsy from infected tissues), are also diagnostic if they lead to the isolation of MAC organisms or show granulomata on histopathologic examination. Should culture be negative despite an high degree of clinical suspicion, it must be repeated in order to achieve an etiological diagnosis. Finally, tuberculosis must be excluded in all cases of suspected MAC, especially when pulmonary infiltrates and acid-fast bacilli smear positivity are recognized. With regard to patients with advanced HIV infection, systematic culture search of MAC from peripheral blood is a sensitive and easy method for diagnosing disseminated MAC infection. Mycobacterial blood cultures allow to establish the diagnosis of disseminated MAC disease with a very good sensitivity (86\% to $98 \%$ of cases in which disseminated MAC infection was subsequently confirmed at necropsy examination) $(28,67)$. While one blood culture leads to identify around $90 \%$ of HIV-infected patients with MAC bacteremia, the adjunct of a second blood culture increases the identification rate up to $98 \%(53,74)$. Blood seeding into liquid and solid media is usually preceded by a lysis of peripheral blood leukocytes, to release intracellular mycobacteria (Isolator System) $(23,54)$. Using liquid cultu- re media, MAC can be detected from blood cultures in as few as 6-12 days, whereas 15-40 days are required with the traditional solid media. Furthermore, currently available DNA probes and reverse hybridisation PCR assays can identify and distinguish among MAC species within as few hours, after that a sufficient mycobacterial growth has occurred in culture media $(23,39)$. In addition, tissue biopsies obtained from normally sterile body sites are also diagnostic. For example, histological stain of biopsy specimens obtained from bone marrow, lymph nodes, or liver showing acidfast bacilli or tuberculous-like granulomata, may be observed several weeks before blood culture results become available $(42,43)$. It is also possible that cultures of bone marrow, lymph node, or liver, could be more sensitive in comparison with blood cultures, especially when a chronic disease has been already established, and a deep immunodeficiency occur.

\section{Therapeutic management of MAC infection in the setting of HIV disease}

The treatment of disseminated MAC disease is seldom straightforward, especially in the field of HIV infection and AIDS. Diagnostic uncertainty is common, prolonged durations of therapy are frequently needed, and eradication is unlikely. Moreover, treatment regimens are expensive, and are often poorly tolerated because of frequent side effects, while the drug-drug interaction with multiple compounds frequently co-administered frequently affects patient with HIV disease (12). As known, NTM are not killed by standard antituberculous drugs at in vivo achievable plasma concentrations with the exception of ethambutol. However, based on extensive observational in vitro testing, at least $50 \%$ of MAC strains can be inhibited by achievable serum levels of some antimicrobial drugs, including rifabutin, rifampin, clofazimine, cycloserine, amikacin, ethionamide, azithromycin, clarithromycin, ciprofloxacin, sparfloxacin, and moxifloxacin. Unfortunately, plasma drug levels needed to obtain a complete in vitro killing of MAC (the so-called minimum bactericidal concentration, or MBC value) range from 8 to $\geq 32$ times compared with minimum inhibitory levels (minimum inhibitor concentration, or MIC value) (76). While associated antimycobacterial agents have shown in vitro inhibitory synergism, a truly bactericidal synergism has been more difficult to be demonstrated (22, 34). Moreover, in order to ensure in vivo bactericidal activity, drugs must penetrate mononuclear cells (especially macrophages) at a suitable concentrations, and must ensure levels capable to pass the bacterial cell wall of MAC organisms. Anyway, some relia- 
ble animal models of disseminated MAC infection showed that both single and combination antimycobacterial regimens led to reduced mycobacterial colony counts by several logs, and also improved survival (22, 34). Medical therapy for disseminated MAC disease involves multiple agents, in order to facilitate clearance of organisms, and to minimize drug resistances. The cornerstone agents include macrolides, ethambutol, and the rifamycin derivative rifabutin (12). Three-drug regimens are preferred because of concerns for the development of macrolides resistance. In fact, some last-generation macrolides, in particular clarithromycin and azithromycin, showed in vitro and in vivo efficacy against MAC. A multicenter, randomized, placebo-controlled trial performed in the pre-cART era, assessed clarithromycin monotherapy, in dosages of 500, 1000, and $2000 \mathrm{mg}$ (all twice daily), in patients with a primary diagnosis of disseminated MAC. The investigators reported a median decrease of over 2 logs in bacterial colony-forming units from blood culture specimens, thus demonstrating a more potent microbiologic effect than had been reported in earlier trials, together with an affordable in vivo response (10). However, a dose-response effect was not observed, but elevated gastrointestinal intolerance occurred with the 2000-mg dosage. Although the 1000-mg twice-daily dosage led to a greater microbiologic response versus the 500-mg twice-daily dosing, there was a trend towards an increased mortality in association with the 1000-mg dosage (10). It was not surprising that after 2-3 months of macrolide administration (regardless of dosages), drug resistance emerged involving about $50 \%$ of patients. Although daily therapy is strongly recommended, for patients who cannot tolerate daily therapy or those who do not require an aggressive treatment strategy, intermittent (three times-weekly) therapy may be adequate. Macrolides administration should be combined with at least another antimycobacterial agent, in order to prevent or delay the emergence of resistance, which is strongly associated with clinical deterioration, and a less favourable outcome. Another study confirmed that the 1000-mg twicedaily dosage of clarithromycin has an adverse effect on survival compared with the $500 \mathrm{mg}$ twice-daily dosing (15). In a trial of azithromycin monotherapy, patients with newly diagnosed positive MAC blood cultures received randomized administration of 1 of 2 doses of azithromycin, 600 or 1200 mg daily. After six weeks, approximately one half of blood cultures were sterile in both groups, with a mean reduction in mycobacteremia of 2 and 1.55 logs, respectively (40). As already observed with clarithromycin, the higher dosage of azithromycin was related to a higher rate of gastrointestinal intolerance. A number of other antimycobacterial agents has been assessed in several randomized, controlled trials. In one trial performed before the introduction of cART, investigators compared the microbiologic efficacy of four-week monotherapy regimens of rifampin, ethambutol, or clofazimine in patients with previously untreated disseminated MAC (38): only ethambutol achieved a statistically significant reduction in blood MAC colony-forming units, suggesting that ethambutol might be the most potent of these three agents. A rifampin derivative, rifabutin, had been specifically developed for the management of disseminated MAC infection in the immunocompromised patient (38). In an early randomized, placebo-controlled trial in which patients with newly diagnosed disseminated MAC disease were blindly assigned to receive clofazimine/ethambutol or clofazimine/ethambutol/rifabutin (at $600 \mathrm{mg} /$ day), approximately half of the patients receiving the rifabutin-containing regimen had a $>2$ log decrease in blood MAC colony-forming units, or sterilization of the blood, compared with none of those receiving only clofazimine/ethambutol combination (59). The long-term clinical outcome of combination regimens including both macrolide and non-macrolide agents for treatment of disseminated HIV-associated MAC disease, was confirmed in a randomized multicenter trial published in 1996 (55). This study included 187 patients with ascertained MAC mycobacteremia, who were randomized to receive clarithromycin at 1,000 mg twice daily, rifabutin 300-600 mg once daily, and ethambutol $15 \mathrm{mg} / \mathrm{kg} /$ day, or a regimen of ciprofloxacin $750 \mathrm{mg}$ twice daily, rifampin 600 mg once daily, clofazimine $100 \mathrm{mg}$ once daily, and ethambutol $15 \mathrm{mg} / \mathrm{kg} /$ day (55) The in vivo quantitative antimycobacterial efficacy tested significantly better with the macrolide-containing regimen, as was median patients' survival (8.6 versus 5.2 months; $p<.001$ ). Although multiple investigations contributed with the evidence of positive results, the composition of the optimal treatment regimen for disseminated MAC in patients with HIV disease is still uncertain. Based on the available microbiological and clinical data, a macrolide drug (either clarithromycin or azithromycin), should be part of the regimen. No significant differences in mortality rates was noticed in a randomized, double-blind trial comparing azithromycin at $600 \mathrm{mg}$ once daily with clarithromycin $500 \mathrm{mg}$ orally twice a day, in an ethambutol-based regimen (20). Evidences coming from a trial of several clarithromycin-containing regimens suggested that clofazimine compared poorly with ethambutol as a second drug added to clarithromycin (18). Furthermore, a randomized clinical trial that added 
clofazimine to clarithromycin plus rifabutin found a greater mortality rate in the clofazimine-containing arm (62\% with clofazimine versus 38\% without clofazimine; $p=.012$ ) (11). Another randomized trial which assessed a four-drug, oral antimycobacterial regimen with or without amikacin showed no clinical benefit associated with the additional amikacin schedule (49). A large, openlabel, prospective, randomized trial comparing the efficacy and safety of clarithromycin plus ethambutol, rifabutin, or both for the treatment of disseminated MAC was conducted in 2003 (6). Of the 203 enrolled patients, 53 received clarithromycin and ethambutol, 50 received clarithromycin and rifabutin, and 57 received all three drugs. A complete microbiologic response (as defined by sterile blood cultures at 12 weeks), was found in $40 \%$, $42 \%$ and $51 \%$ of cases, respectively. Notably, a significant improvement in survival was observed in the three-drug arm. Anyway, another randomized, placebo-controlled study which assessed the addition of rifabutin to a regimen of clarithromycin and ethambutol did not find any difference in the microbiologic response or survival benefit between the two stated arms (24). Moreover, this last study detected a significant reduction in the development of clarithromycin resistance in the arm containing three drugs ( $2 \%$ versus $14 \%$ ), thus suggesting a benefit in the field of microbial resistance. Macrolide resistance occurs during macrolide monotherapy, although the concomitant use of fluoroquinolones has been suggested as an adjunctive supporting cause. Although both fluoroquinolones and carbapenems have been reported to be effective in vitro and in vivo against a broad spectrum of NTM $(17,68,78)$, the combination usage does not allow to distinguish the contribution of each single agent, when in vivo therapeutic courses are assessed (17). Regimens that do not include ethambutol are also associated with the development of drug resistance. Unfortunately, in cases of macrolide resistance, mortality occurs within one year in approximately one third of patients and, nearly half of patients will die within two years (12). In the immunocompromised host, treatment should be continued for up to 12 months; negative sputum cultures should be obtained in pulmonary localizations. Therefore, the typical duration of treatment may range from 18 to 24 months, but it can be even longer for some individuals, whose immune recovery is slow. Also in the cART era, treatment failure and recurrences of disseminated MAC disease may occur. Patients are considered to have a treatment failure if they did not show clinical improvements after six months of appropriate treatment, or did not achieve negative sputum culture after 12 months of appropriate therapy (in the event of isolated respiratory tract disease).

Treatment failure may be related to non-compliance or intolerance, local anatomic defects (cavitations or bronchiectasis, in case of lung disease), or drug resistance (especially to macrolides). In selected cases of disseminated NTM infections (i.e. the forms related to the so-called immune reconstitution syndrome occurring in AIDS patients effectively treated with antiretroviral therapy), steroid adjunct may act favourably (58, 62, 63). Relapses and re-infections are also common, and may not be necessarily related to drug resistance. Patients showing treatment failures are unlikely to have a successful course of re-treatment. Therefore many authors advocate aggressive treatment initially. Surgical resection may be effective in patients with focal (especially pulmonary) MAC disease, and it is considered the treatment of choice for solitary pulmonary nodules.

\section{Antimycobacterial prophylaxis guidelines}

Since up to $40 \%$ of patients with advanced HIV disease are likely to develop disseminated MAC, it makes sense to develop a strategy for preventing this disease in patients at risk (the so-called primary prophylaxis). Few specific risk factors, other than a low CD4+ lymphocyte count, have been clearly defined until now. Therefore, any current prophylactic strategy must be applied to the entire population at risk including patients with HIV infection and a CD4+ count $<50$ cells $/ \mu \mathrm{L}$. Results of combined analysis of two randomized, placebocontrolled trials of rifabutin prophylaxis carried out in the pre-cART era, which included more than 1,000 patients with advanced HIV disease, showed that rifabutin at $300 \mathrm{mg} /$ day reduced the incidence of mycobacteremia by about 50\% (45). Interestingly, patients who received rifabutin and subsequently developed mycobacteremia, had blood MAC isolates that retained susceptibility to rifabutin. However, neither each single trial, nor their combined analysis, demonstrated that rifabutin significantly reduced mortality. Combined analysis of both trials revealed an increased incidence of fever, fatigue, anaemia, elevated alkaline phosphatase levels, and hospitalizations, in patients who received placebo versus those who received rifabutin. Patients assigned to the placebo arm in these last trials, however, tended to have a lower absolute CD4+ count, which may have accounted for some of the greater morbidity (45). A further placebo-controlled study provided the first evidence that MAC prophylaxis could improve survival of patients with late-stage HIV disease. Six hundred and 82 patients with advanced HIV disease were randomly assigned to receive either clarithromycin (500 mg) or placebo twice daily. During a 
median 10-month follow-up period, only $6 \%$ of clarithromycin-treated subjects developed mycobacteremia compared with $16 \%$ of placebo-assigned patients $(p<.001)$ (51). Notably, the median patients' survival time was significantly longer for clarithromycin-assigned patients than for placeboassigned patients (8.6 versus 5.2 months; $p=.001$ ). Dose-limiting drug toxicity occurred in $8 \%$ of the clarithromycin group, and $6 \%$ of the placebo group. A prior trial had already provided even more convincing evidence of the long-term benefit of clarithromycin prophylaxis for MAC in patients with very advanced HIV disease. A total of 1,216 patients with a median absolute CD4+ lymphocyte count of 28 cells/ $\mu \mathrm{L}$ were randomly assigned to receive rifabutin (300-450 mg/day), clarithromycin (500 mg twice daily), or their association (5). Median follow-up of patients enrolled in this trial was 589 days (over 19 months), longer than in any other MAC prophylaxis trial ever performed; overall survival was approximately $50 \%$. At the conclusion of the trial, deaths in the respective groups were: clarithromycin, $42 \%$; rifabutin, $43 \%$; and the combination, $46 \%$. In an intent-to-treat analysis, only $9 \%$ of patients assigned to clarithromycin developed disseminated MAC, compared with $15 \%$ assigned to rifabutin $(p<.001)$. In a third macrolide prophylaxis trial, 693 patients with less than $100 \mathrm{CD} 4+$ cells/ $\mu \mathrm{L}$ were randomly assigned azithromycin (1,200 mg once weekly), rifabutin (300 mg/day), or a combination of these drugs (27). During the median follow-up time of 514 days, the incidence of disseminated MAC proved lower in the azithromycin group (13.9\%) or the combination group (8.3\%) versus that one observed in subjects receiving rifabutin only (23.3\%). Dose-limiting drug toxicity occurred in 13\%, 23\%, and $16 \%$ of the three stated patient groups, respectively (27). Interestingly, the occurrence of macrolide-resistant MAC disease has been an infrequent event in the reported prophylaxis studies: only 2$3 \%$ of patients given clarithromycin and only $1 \%$ of patients given azithromycin suffered from an mycobacteremia with MAC isolates resistant to these drugs. In the 2000, two randomized, controlled studies have compared azithromycin with placebo in patients with a CD4+ count increased to at least 100 cells/ $\mu \mathrm{L}$ while on a potent cART treatment, and no prior history of disseminated MAC disease (13). In a study, a total of 520 patients were enrolled with a median CD4+ count of 230 cells $/ \mu \mathrm{L}$ at the time of entry. There were no cases of confirmed MAC in either the azithromycin arm or the placebo arm over a 12-month observation period (11). In another study, which enrolled 643 patients, only two episodes of MAC disease were observed among the 321 patients assigned to pla- cebo, and no cases of MAC occurred among patients who remained on azithromycin, leading to an estimated incidence of 0.5 events per 100 person-years (21). In both of these studies, the discontinuation of azithromycin prophylaxis was not found to increase the incidence of MAC disease during a median of 12-16-month follow-up, suggesting that it is safe to discontinue primary prophylaxis in those patients who achieved a significant CD4+ recovery attributed to an effective cART regimen (21). With regard to secondary prophylaxis against MAC, in the earliest study evaluating the safety of discontinuing secondary prophylaxis for disseminated MAC, the investigators prospectively followed four patients with a known history of MAC disease. Antimycobacterial therapy was discontinued without evidence of recurrence after 8-13 months of follow-up (2). Later, in a larger, prospective trial, a total of 48 patients discontinued antimycobacterial therapy (1). All patients had been on a macrolide-based regimen for at least 12 months, and on cART obtaining a CD4+ count of at least 100 cells $/ \mu \mathrm{L}$, sustained for at least 16 weeks. Of the 48 subjects, 47 remained free from MAC complications for a median of 77 weeks off therapy. One patient developed a localized MAC osteomyelitis 16 months after stopping secondary prophylaxis (1). Concurrently, in a French retrospective study, three patients of 26 experienced a MAC relapse (80): one of them was extremely immunocompromised (his CD4+ count was $<50$ cells/ $\mu \mathrm{L}$ at the time of relapse), and the other two individuals developed atypical bone infections, concurrently with a CD4+ lymphocyte count of 126 and 160 cells $/ \mu \mathrm{L}$, respectively (80). In patients with increased CD4+ counts on cART, who develop atypical manifestations of MAC, it may be prudent to continue treatment for a prolonged period of 12-18 months, and then continue chronic suppressive therapy (secondary prophylaxis), for life. It is also critical to remember to re-start MAC prophylaxis if immunologic failure and/or viral load rising result in a decrease in the CD4+ count. Presently, the absolute CD4+ cell threshold indicating re-initiation is still debated, although the US Public Health Service guidelines recommend to consider a CD4+ lymphocyte count $<50$ cells/ $\mu \mathrm{L}$, as a risk threshold to start antimicrobial prophylaxis. To conclude, in the cART era it appears safe to discontinue antimycobacterial therapy in patients who have been successfully treated their underlying HIV infection, and achieved a sustained CD4+ lymphocyte count $>100$ cells $/ \mu \mathrm{L}$ for at least three months, and were previously treated with a macrolide-containing therapeutic regimen for at least 12 months. 


\section{REFERENCES}

1. Aberg JA, Williams PL, Liu T, et al. A study of discontinuing maintenance therapy in human immunodeficiency virus-infected subjects with disseminated Mycobacterium avium complex. J Infect Dis 2003; 187: 1046-52.

2. Aberg JA, Yajko DM, Jacobson MA. Eradication of AIDS-related disseminated Mycobacterium avium complex infection after 12 months of antimycobacterial therapy combined with highly active antiretroviral therapy. J Infect Dis 1998; 178: 1446-9.

3. Alisky JM, Schlesinger L. Isolated cavitary pulmonary Mycobacterium avium complex infection in a patient with AIDS. Clin Infect Dis 1998; 27: 1542-3.

4. Appelberg R, Castro AG, Pedrosa J, Silva RA, Orme IM, Minoprio P. Role of gamma interferon and tumor necrosis factor alpha during T-cell-independent and dependent phases of Mycobacterium avium infection. Infect Immun 1994; 62: 3962-71.

5. Benson CA, Williams PL, Cohn DL, et al. Clarithromycin or rifabutin alone or in combination for primary prophylaxis of Mycobacterium avium complex disease in patients with AIDS: A randomized, double-blind, placebo-controlled trial. J Infect Dis 2000; 181: 1289-97.

6. Benson CA, Williams PL, Currier JS, et al. A prospective, randomized trial examining the efficacy and safety of clarithromycin in combination with ethambutol, rifabutin, or both for the treatment of disseminated Mycobacterium avium complex disease in persons with acquired immunodeficiency syndrome. Clin Infect Dis 2003; 37: 1234-43.

7. Blumenthal DR, Zucker JR, Hawkins CC. Mycobacterium avium complex-induced septic arthritis and osteomyelitis in a patient with the acquired immunodeficiency syndrome. Arthritis Rheum 1990; 33: 757-8.

8. Cappell MS, Gupta A. Gastrointestinal hemorrhage due to gastrointestinal Mycobacterium avium intracellulare or esophageal candidiasis in patients with the acquired immunodeficiency syndrome. Am J Gastroenterol 1992; 87: 224-9.

9. Castro AG, Silva RA, Appelberg R. Endogenously produced IL-12 is required for the induction of protective T cells during Mycobacterium avium infections in mice. J Immunol 1995; 155: 2013-9.

10. Chaisson RE, Benson CA, Dubé MP, et al. Clarithromycin therapy for bacteremic Mycobacterium avium complex disease. A randomized, double-blind, dose-ranging study in patients with AIDS. Ann Intern Med 1994; 121: 905-11.

11. Chaisson RE, Keiser $\mathrm{P}$, Pierce $\mathrm{M}$, et al. Clarithromycin and ethambutol with or without clofazimine for the treatment of bacteremic Mycobacterium avium complex disease in patients with HIV infection. AIDS 1997; 11: 311-7.

12. Chaisson RE, Moore RD, Richman DD, Keruly J, Creagh $T$. Incidence and natural history of Mycobacterium avium-complex infections in patients with advanced human immunodeficiency virus disease treated with zidovudine. The Zidovudine Epidemiology Study Group. Am Rev Respir Dis 1992; 146: 285-9.

13. Chin DP, Hopewell PC, Yajko DM, et al. Mycobacterium avium complex in the respiratory or gastrointestinal tract and the risk of $M$. avium complex bacteremia in patients with human immunodeficiency virus infection. J Infect Dis 1994; 169: 289-95.

14. Cohen JI, Saragas SJ. Endophthalmitis due to Mycobacterium avium in a patient with AIDS. Ann
Ophthalmol 1990; 22: 47-51.

15. Cohn DL, Fisher EJ, Peng GT, et al. A prospective randomized trial of four three-drug regimens in the treatment of disseminated Mycobacterium avium complex disease in AIDS patients: excess mortality associated with high-dose clarithromycin. Clin Infect Dis 1999; 29: 125-33.

16. Crawford JT, Bates JH. Analysis of plasmids in Mycobacterium avium-intracellulare isolates from persons with acquired immunodeficiency syndrome. Am Rev Respir Dis 1986; 134: 659-61.

17. Cremades R, Santos A, Rodriguez JC, Garcia-Pachon E, Ruiz M, Royo G. In vitro bactericidal activity of antibiotic combinations against clinical isolates of Mycobacterium chelonae. J Chemother 2008; 20: 43-7.

18. Denis M, Gregg EO. Recombinant tumour necrosis factor-alpha decreases whereas recombinant interleukin-6 increases growth of a virulent strain of Mycobacterium avium in human macrophages. Immunology 1990; 71: 139-41.

19. Dubé MP, Sattler FR, Torriani FJ, et al. A randomized evaluation of ethambutol for prevention of relapse and drug resistance during treatment of Mycobacterium avium complex bacteremia with clarithromycin-based combination therapy. California Collaborative Treatment Group. J Infect Dis 1997; 176: 1225-32.

20. Dunne M, Fessel J, Kumar P, et al. A randomized, double-blind trial comparing azithromycin and clarithromycin in the treatment of disseminated Mycobacterium avium infection in patients with human immunodeficiency virus. Clin Infect Dis 2000; 31: 1245-52.

21. El-Sadr WM, Burman WJ, Grant LB, et al. Discontinuation of prophylaxis for Mycobacterium avium complex disease in HIV-infected patients who have a response to antiretroviral therapy. $N$ Engl $J$ Med 2000; 342: 1085-92.

22. Fernandes PB, Hardy DJ, McDaniel D, Hanson CW, Swanson RN. In vitro and in vivo activities of clarithromycin against Mycobacterium avium. Antimicrob Agents Chemother 1989; 33: 1531-4.

23. Gazzola L, Zanini F, Zerbi P, Franzetti F, Gori A. The usefulness of PCR assay in diagnosing disseminated mycobacterial infection in AIDS patients. Eur J Clin Microbiol Infect Dis 2008; 27: 163-6.

24. Gordin FM, Sullam PM, Shafran SD, et al. A randomized, placebo-controlled study of rifabutin added to a regimen of clarithromycin and ethambutol for treatment of disseminated infection with Mycobacterium avium complex. Clin Infect Dis 1999; 28: 1080-5.

25. Griffith DE, Aksamit T, Brown-Elliott BA, et al. An official ATS/IDSA statement: diagnosis, treatment, and prevention of nontuberculous mycobacterial diseases. Am J Respir Crit Care Med 2007; 175: $367-$ 416

26. Hampson SJ, Portaels F, Thompson J, et al. DNA probes demonstrate a single highly conserved strain of Mycobacterium avium infecting AIDS patients. Lancet 1989; 1: 65-8.

27. Havlir DV, Dube MP, Sattler FR, et al. Prophylaxis against disseminated Mycobacterium avium complex with weekly azithromycin, daily rifabutin, or both. $N$ Engl J Med 1996; 335: 392-8.

28. Hawkins CC, Gold JW, Whimbey E, et al. Mycobacterium avium complex infections in patients with the acquired immunodeficiency syndrome. Ann Intern Med 1986; 105: 184-8.

29. Hocqueloux L, Lesprit P, Herrmann JL, et al. Pulmonary Mycobacterium avium complex disease without dissemination in HIV-infected patients. Chest 1998; 113: 542-8. 
30. Horsburgh CR, Jr., Cohn DL, Roberts RB, et al. Mycobacterium avium-M. intracellulare isolates from patients with or without acquired immunodeficiency syndrome. Antimicrob Agents Chemother 1986; 30: 955-7.

31. Horsburgh CR, Jr., Mason UG, 3rd, Farhi DC, Iseman MD. Disseminated infection with Mycobacterium avium-intracellulare. A report of 13 cases and a review of the literature. Medicine (Baltimore) 1985; 64: 36-48.

32. Horsburgh CR, Jr., Selik RM. The epidemiology of disseminated nontuberculous mycobacterial infection in the acquired immunodeficiency syndrome (AIDS). Am Rev Respir Dis 1989; 139: 4-7.

33. Howard ST, Rhoades E, Recht J, et al. Spontaneous reversion of Mycobacterium abscessus from a smooth to a rough morphotype is associated with reduced expression of glycopeptidolipidic and reacquisition of an invasive phenotype. Microbiology 2006; 152: 1581-90.

34. Inderlied CB, Kolonoski PT, Wu M, Young LS. Amikacin, ciprofloxacin, and imipenem treatment for disseminated Mycobacterium avium complex infection of beige mice. Antimicrob Agents Chemother 1989; 33: 176-80.

35. Iseman MD, Buschman DL, Ackerson LM. Pectus excavatum and scoliosis. Thoracic anomalies associated with pulmonary disease caused by Mycobacterium avium complex. Am Rev Respir Dis 1991; 144: 914-6.

36. Jacobson MA, Hopewell PC, Yajko DM, et al. Natural history of disseminated Mycobacterium avium complex infection in AIDS. J Infect Dis 1991; 164: 994-8.

37. Kansal RG, Gomez-Flores E, Mehta RT. Change in colony morphology influences the virulence as well as the biochemical properties of the Mycobacterium avium complex. Microb Pathog 1998; 25: 203-14.

38. Kemper CA, Havlir D, Haghighat D, et al. The individual microbiologic effect of three antimycobacterial agents, clofazimine, ethambutol, and rifampin, on Mycobacterium avium complex bacteremia in patients with AIDS. J Infect Dis 1994; 170: 157-64.

39. Kiehn TE, Edwards FF. Rapid identification using a specific DNA probe of Mycobacterium avium complex from patients with acquired immunodeficiency syndrome. J Clin Microbiol 1987; 25: 1551-2.

40. Koletar SL, Berry AJ, Cynamon MH, et al. Azithromycin as treatment for disseminated Mycobacterium avium complex in AIDS patients. Antimicrob Agents Chemother 1999; 43: 2869-72.

41. Miller RS, Thomas SJ, Hospenthal DR, Oster CN. Isolated Mycobacterium avium complex osteomyelitis in a patient with AIDS. In: Program and Abstracts of the 35th Annual Meeting of the Infectious Diseases Society of America; September 13-16, 1997; San Francisco. Abstract 574.

42. Newman GW, Guarnaccia JR, Vance EA, Wu JY, Remold HG, Kazanjian PH. Interleukin-12 enhances antigen-specific proliferation of peripheral blood mononuclear cells from HIV-positive and negative donors in response to Mycobacterium avium. AIDS 1994; 8: 1413-9.

43. Nichols L, Florentine B, Lewis W, Sattler F, Rarick MU, Brynes RK. Bone marrow examination for the diagnosis of mycobacterial and fungal infections in the acquired immunodeficiency syndrome. Arch Pathol Lab Med 1991; 115: 1125-32.

44. Nightingale SD, Byrd LT, Southern PM, Jockusch JD, Cal SX, Wynne BA. Incidence of Mycobacterium avium-intracellulare complex bacteremia in human immunodeficiency virus-positive patients. J Infect Dis 1992; 165: 1082-5.
45. Nightingale SD, Cameron DW, Gordin FM, et al. Two controlled trials of rifabutin prophylaxis against Mycobacterium avium complex infection in AIDS. $N$ Engl J Med 1993; 329: 828-33.

46. Northfelt DW, Mayer A, Kaplan LD, et al. The usefulness of diagnostic bone marrow examination in patients with human immunodeficiency virus (HIV) infection. J Acquir Immune Defic Syndr 1991; 4: 65966.

47. Nyberg DA, Federle MP, Jeffrey RB, Bottles K, Wofsy CB. Abdominal CT findings of disseminated Mycobacterium avium-intracellulare in AIDS. Am J Roentgenol 1985; 145: 297-9.

48. Palella FJ, Delaney KM, Moorman AC, et al. Declining morbidity and mortality among patients with advanced human immunodeficiency virus infection. HIV Outpatient Study Investigators. $N$ Engl $J$ Med 1998; 338: 853-60.Parenti DM, Williams PL, Hafner R, et al. A phase II/III trial of antimicrobial therapy with or without amikacin in the treatment of disseminated Mycobacterium avium infection in HIVinfected individuals. AIDS 1998; 12: 2439-46.

49. Phillips P, Kwiatkowski MB, Copland M, Craib K, Montaner J. Mycobacterial lymphadenitis associated with the initiation of combination antiretroviral therapy. J Acquir Immune Defic Syndr Hum Retrovirol 1999; 20: 122-8.

50. Pierce M, Crampton S, Henry D, et al. A randomized trial of clarithromycin as prophylaxis against disseminated Mycobacterium avium complex infection in patients with advanced acquired immunodeficiency syndrome. N Engl J Med 1996; 335:384-91.

51. Race EM, Adelson-Mitty J, Kriegel GR, et al. Focal mycobacterial lymphadenitis following initiation of protease-inhibitor therapy in patients with advanced HIV-1 disease. Lancet 1998; 351: 252-5.

52. Reves R, Stone-Venohr B, Hildred G, et al. Utility of paired blood cultures in the diagnosis of disseminated Mycobacterium avium complex infection (abstract). In: Proceedings of the 8th International Conference on AIDS, Amsterdam, July 19-24, 1992. Amsterdam: Dutch Foundation 1992; 127.

53. Roberts GD, Koneman EW, Kin YK. Mycobacterium. In: Balows A, Hausler WJ Jr, Herrmann KL, et al., eds. Manual of Clinical Microbiology. Washington, DC: American Society of Microbiology, 1991; 304339.

54. Shafran SD, Singer J, Zarowny DP, et al. A comparison of two regimens for the treatment of Mycobacterium avium complex bacteremia in AIDS: rifabutin, ethambutol, and clarithromycin versus rifampin, ethambutol, clofazimine, and ciprofloxacin. N Engl J Med 1996; 335: 377-83.

55. Shanson DC, Dryden MS. Comparison of methods for isolating Mycobacterium avium-intracellulare from blood of patients with AIDS. J Clin Pathol 1988; 41: 687-90.

56. Sheppard DC, Sullam PM. Primary septic arthritis and osteomyelitis due to Mycobacterium avium complex in a patient with AIDS. Clin Infect Dis 1997; 25: 925-6.

57. Streit M, Böhlen LM, Hunziker T, et al. Disseminated Mycobacterium marinum infection with extensive cutaneous eruption and bacteremia in an immunocompromised patient. Eur J Dermatol 2006; 16: 79-83.

58. Sullam PM, Gordin FM, Wynne BA. Efficacy of rifabutin in the treatment of disseminated infection due to Mycobacterium avium complex. The Rifabutin Treatment Group. Clin Infect Dis 1994; 19: 84-6.

59. Tortoli E, Rindi L, Garcia MJ., et al. Proposal to elevate the genetic variant MAC-A included in the Mycobacterium avium complex, to species rank as 
Mycobacterium chimerae sp. nov. Int J System Evol Microbiol 2004; 54: 1277-85.

60. Tsang AY, Denner JC, Brennan PJ, McClatchy JK. Clinical and epidemiological importance of typing of Mycobacterium avium complex isolates. J Clin Microbiol 1992; 30: 479-84.

61. Tuon FF, Mulatti GC, Pinto WP, De Siqueira Franca FO, Gryschek RC. Immune reconstitution inflammatory syndrome associated with disseminated mycobacterial infection in patients with AIDS. AIDS Patient Care STDs 2007; 21: 527-32.

62. Vemulapalli RK, Cantey JR, Steed LL, Knapp TL, Thielman NM. Emergence of resistance to clarithromycin during treatment of disseminated cutaneous Mycobacterium chelonae infection: case report and literature review. J Infect 2001; 43: 163-8.

63. Volpe F, Schwimmer A, Barr C. Oral manifestation of disseminated Mycobacterium avium intracellulare in a patient with AIDS. Oral Surg Oral Med Oral Pathol 1985; 60: 567-70.

64. von Reyn CF, Barber TW, Arbeit RD, et al. Evidence of previous infection with Mycobacterium aviumMycobacterium intracellulare complex among healthy subjects: an international study of dominant mycobacterial skin test reactions. J Infect Dis 1993; 168: 1553-8.

65. von Reyn CF, Maslow JN, Barber TW, Falkinham JO, Arbeit RD. Persistent colonisation of potable water as a source of Mycobacterium avium infection in AIDS. Lancet 1994; 343: 1137-41.

66. Wallace JM, Hannah JB. Mycobacterium avium complex infection in patients with the acquired immunodeficiency syndrome. A clinicopathologic study. Chest 1988; 93: 926-32.

67. Watt B, Edwards JR, Rayner A, Grindey AJ, Harris G. In vitro activity of meropenem and imipenem against mycobacteria: development of a daily antibiotic dosing schedule. Tuber Lung Dis 1992; 73: 134-6.

68. Wayne LG, Young LS, Bertram M. Absence of mycobacterial antibody in patients with acquired immune deficiency syndrome. Eur J Clin Microbiol 1986; 5: 363-5.

69. Winter SM, Bernard EM, Gold JW, Armstrong D.
Humoral response to disseminated infection by Mycobacterium avium-Mycobacterium intracellulare in acquired immunodeficiency syndrome and hairy cell leukemia. J Infect Dis 1985; 151: 523-7.

70. Wolinsky E. Nontuberculous mycobacteria and associated diseases. Am Rev Respir Dis 1979; 119: 107-59.

71. Wong B, Edwards FF, Kiehn TE, et al. Continuous high-grade Mycobacterium avium-intracellulare bacteremia in patients with the acquired immune deficiency syndrome. Am J Med 1985; 78: 35-40.

72. Woods GL, Goldsmith JC. Fatal pericarditis due to Mycobacterium avium-intracellulare in acquired immunodeficiency syndrome. Chest 1989; 95: 13557.

73. Yagupsky P, Menegus MA. Cumulative positivity rates of multiple blood cultures for Mycobacterium avium-intracellulare and Cryptococcus neoformans in patients with the acquired immunodeficiency syndrome. Arch Pathol Lab Med 1990; 114: 923-5.

74. Yajko DM, Kirihara J, Sanders C, Nassos P, Hadley WK. Antimicrobial synergism against Mycobacterium avium complex strains isolated from patients with acquired immune deficiency syndrome. Antimicrob Agents Chemother 1988; 32: 1392-5.

75. Yajko DM, Nassos PS, Hadley WK. Therapeutic implications of inhibition versus killing of Mycobacterium avium complex by antimicrobial agents. Antimicrob Agents Chemother 1987; 31: 117-20.

76. Yakrus MA, Good RC. Geographic distribution, frequency, and specimen source of Mycobacterium avium complex serotypes isolated from patients with acquired immunodeficiency syndrome. J Clin Microbiol 1990; 28: 926-9.

77. Yew WW, Lau KS, Tse WK, Wong CF. Imipenem in the treatment of lung infections due to Mycobacterium fortuitum and Mycobacterium chelonae: further experience. Clin Infect Dis 1992; 15: 1046-7

78. Zakowski P, Fligiel S, Berlin GW, Johnson L, Jr. Disseminated Mycobacterium avium-intracellulare

79. Zeller V, Truffot C, Agher R, et al. Discontinuation of secondary prophylaxis against disseminated Mycobacterium avium complex infection and toxoplasmic encephalitis. Clin Infect Dis 2002; 34: 662-7 